\title{
The Hydrogen Atom from a New Angle
}

\author{
Valeri Tomashau ${ }^{1^{*}}$ \\ ${ }^{1}$ LC Engineers, Inc., 1471 Pinewood Street, Rahway, NJ 07065, U.S.A. \\ * Corresponding author. Tel.: 732-379-9691; email: vtomashev@gmail.com \\ Manuscript submitted June 28, 2014; accepted September 12, 2014. \\ doi: 10.7763/IJAPM.2014.V4.303
}

\begin{abstract}
Turning back to Niels Bohr's model of the hydrogen atom, the paper is trying to resolve one of the issues associated with the model. While N. Bohr in the framework of his model did not find any relation between electron's motion in the atom and radiation emitted, there is a reason to believe that the relation is bound to exist. Furthermore, it is already given by Rydberg's formula that expresses the frequency of radiation as a difference of two other frequencies associated in some manner with electron's rotation.

Based on this approach a new value for the radius of the ground stationary orbit is calculated. It appears to be more than Bohr's radius by a factor of 1.588. The question why both values for the radius of the ground stationary orbit (Bohr's radius and a new one) are compatible with Rydberg's formula is considered. Finally, what exactly Niels Bohr overlooked in his model of the hydrogen atom, is discussed. It is established that Rydberg's formula can be derived from the more general parameterized postulates, when Niels Bohr used, regardless of the value of the parameter.
\end{abstract}

Key words: Simple relation between the motion and radiation, new radius of the first stationary orbit, inaccuracy of Bohr's hydrogen atom model.

\section{Introduction}

In spite of 100-year history, Bohr's hydrogen atom model is still under discussion. There are several unresolved issues in the context of the model, such as:

What is the reason why the stationary orbits do exist and are blurred at the same time?

Why the electron at the stationary orbit does not emit electromagnetic waves?

Where the periodicities, that reveal themselves in the line-spectrum, are hidden in the atom?

The last question is under consideration in this paper. The main idea of the paper is to reveal the relation between the motion of the electron in the atom and the radiation emitted.

Following Rutherford's planetary-model atom [1] and based on regularities that are observed in the line-spectrum of the hydrogen atom Niels Bohr proposed his own model of the atom [2]. The derivation of Bohr's model in contemporary interpretation is widely presented in books [3] and on the Internet [4].

Initially Balmer [5] has found some regularity in visible part of the hydrogen atom spectrum (Balmer's lines) and expressed them by the rather simple formula:

$$
\lambda_{m}=B m^{2} /\left(m^{2}-2^{2}\right),
$$

where $\lambda_{m}$ - wave-length of emitted radiation, $B$ - Balmer's constant, $m=3,4, \ldots$ - ordinal number of electron orbit. Later this empirical formula was generalized and slightly transformed by Rydberg. In 
contemporary notation it appears as shown below:

$$
1 / \lambda_{m n}=R\left(1 / n^{2}-1 / m^{2}\right),
$$

where $R$ - Rydberg's constant, $R \approx 10973731.57 \mathrm{~m}^{-1}(R=4 / B), \quad n=1,2, \ldots, \quad m=2,3,4, \ldots$ - ordinal numbers of electron orbit.

Niels Bohr's postulates-based model gave a new conception of the atomic structure and opened the door for quantum theory. However no relation between the motion of an electron in the atom and radiation emitted has been established in the framework of that model. Nevertheless, the common sense guides us to suppose that the "simple relation between the motion of the atom and the radiation sent out" is bound to exist.

\section{Rydberg's Formula as a Relation between Motion and Radiation}

Strange as it may seems, however the relation between the motion of the atom and the radiation is already given by Rydberg's formula (2) re-written for frequencies $\left(v_{m n}=c / \lambda_{m n}\right)$ :

$$
v_{m n}=R c\left(1 / n^{2}-1 / m^{2}\right), \quad n=1,2, \ldots, \quad m=2,3, \ldots
$$

It expresses the frequency of radiation as a difference of two other frequencies:

$$
v_{m n}=v_{n}-v_{m}
$$

where $v_{\mathrm{m}}=R c / \mathrm{m}^{2}, \quad v_{\mathrm{n}}=R c / \mathrm{n}^{2}$.

What kind of motion in the atom is responsible for these frequencies? Since the main motion in the hydrogen atom is electron's rotation around the proton, it would appear very natural that the rotation of the electron around the nuclear is responsible for these frequencies. To be specific, in the case of the first Lyman's line [6], one frequency $\left(v_{1}=R c / 1^{2}=R c\right)$ is due to electron rotation at the first (ground) stationary orbit, whereas the another one $\left(v_{2}=R c / 2^{2}\right)$ is related, in some manner, to electron rotation at the second stationary orbit. Note that the product of Rydberg's constant $R$ by the velocity of light $c$ has a meaning of frequency of electron rotation at the ground stationary state.

Under the assumption that the frequency $v_{1}$ is produced by the electron rotation at the ground stationary orbit, the radius of this orbit can be easily calculated.

\section{A New Value for Radius of the Ground Stationary Orbit in the Hydrogen Atom}

Since the electron orbits the proton stationary, the electrostatic force of attraction between electron and proton is balanced by centrifugal force:

$$
m_{e} V_{1}^{2} / r_{1}=e^{2} / 4 \pi \varepsilon_{0} r_{1}^{2} \quad==>\quad r_{1}=e^{2} / 4 \pi \varepsilon_{0} \boldsymbol{m}_{e} \boldsymbol{V}_{1}^{2}
$$

where $\boldsymbol{r}_{1}$ - radius of the first (ground) stationary orbit, $\boldsymbol{e}$ - electron's electric charge, $\boldsymbol{\varepsilon}_{0}$ - electric constant, $\boldsymbol{m}_{e}$ - electron's mass, and $\boldsymbol{V}_{1}$ - linear velocity of the electron at the first stationary orbit.

If take in account that $v_{1}=R c / 1^{2}$ and, on the other hand, that $v_{1}=V_{1} / 2 \pi r_{1}$, the expression for $V_{1}^{2}$ can be obtained:

$$
R c=V_{1} / 2 \pi r_{1} \quad==>\quad V_{1}^{2}=(R c)^{2}(2 \pi)^{2} r_{1}^{2}
$$


Through the mediation of substitution (6) to (5) the radius of the first stationary orbit of the hydrogen atom can be expressed in terms of the fundamental constants:

$$
r_{1}^{3}=e^{2} / 2(2 \pi)^{3} \varepsilon_{0} m_{e}(R c)^{2} .
$$

The calculation with the use of the fundamental physical constants [7], such as: Rydberg's constant $\boldsymbol{R} \approx 10973731.57 \mathrm{~m}^{-1}$, electron electric charge $\boldsymbol{e} \approx 1.6022 \times 10^{-19} \mathrm{c}$, electric constant $\boldsymbol{\varepsilon}_{0} \approx 8.8542 \times 10^{-12} \mathrm{~N} \mathrm{~m}^{2} / \mathrm{s}^{2}$, electron mass $\boldsymbol{m}_{\boldsymbol{e}} \approx 9.1094 \times 10^{-31} \mathrm{~kg}$, and velocity of the light $\boldsymbol{c} \approx 2.9979 \times 10^{8} \mathrm{~m} / \mathrm{c}$, gives the value for the radius of the first stationary orbit of the hydrogen atom:

$$
r_{1} \approx 0.840 \times 10^{-10} \mathrm{~m}(0.840 \AA) .
$$

Note that there was no Planck constant among fundamental constants used in the above calculation.

\section{The New First Stationary Orbit Radius vs. Bohr's Radius}

The calculated above value $(0.840 \AA)$ for the radius of the ground stationary orbit of the hydrogen atom differs from Bohr's radius (0.529 $)$ [3], [4] obtained on a basis of Bohr's postulates. However it should be emphasized that our value (8) was derived in natural way from Rydberg's formula comprising with great accuracy experimental facts that have been verified repeatedly. The value of the Rydberg's constant is readily derived from the experimental data and can be considered as an irrefutable experimental fact as well.

On contrary, N. Bohr's value for the radius of the first stationary orbit (Bohr's radius) [3], [4] is calculated under week-grounded assumption that angular moment of electron has to be equal to an integer multiplied by Planck constant (quantization rule - the first Bohr's postulate [2]-[4]):

$$
m_{e} V_{\mathrm{n}} r_{\mathrm{n}}=n h / 2 \pi .
$$

The fact that Planck constant and the angular moment have the same unit of measurement represents a weighty argument, however it would be better to assume the proportionality $m_{e} V_{\mathrm{n}} r_{\mathrm{n}} \sim n h / 2 \pi$ rather than the equality (9) assumed by Niels Bohr.

\section{Electron's Rotation Frequency Dependence of Orbit Number and Effective Frequency}

The well-known $n$-dependences:

$$
r_{\mathrm{n}}=r_{1} n^{2}, \quad V_{\mathrm{n}}=V_{1} / n, \quad T_{\mathrm{n}}=T_{1} n^{3}, \quad \boldsymbol{v}_{\mathrm{n}}=\boldsymbol{v}_{1} / \boldsymbol{n}^{3},
$$

where $n$ is an ordinal number of electron orbit, takes place on the assumption that $m_{e} V_{n} r_{n}=n h / 2 \pi$. As outlined below, they remains valid even though the proportionality $m_{e} V_{\mathrm{n}} r_{\mathrm{n}} \sim n h / 2 \pi$ instead of the above equality is used.

The expression similar to (5) holds for any stationary electron orbit:

$$
m_{e} V_{\mathrm{n}}^{2} / r_{\mathrm{n}}=e^{2} / 4 \pi \varepsilon_{0} r_{\mathrm{n}}^{2} \quad==>\quad \boldsymbol{r}_{\mathrm{n}}=\boldsymbol{e}^{2} / \mathbf{4} \boldsymbol{\pi} \boldsymbol{\varepsilon}_{0} \boldsymbol{m}_{e} \boldsymbol{V}_{\mathrm{n}}^{2},
$$

where $\boldsymbol{r}_{\mathrm{n}}$ - radius of the stationary orbit having number $\boldsymbol{n}, \boldsymbol{e}$ - electron's electric charge, $\boldsymbol{\varepsilon}_{0}-$ electric 
constant, $\boldsymbol{m}_{e}$ - electron's mass, and $\boldsymbol{V}_{\mathrm{n}}$ - linear velocity of the electron at that orbit.

The proportionality $m_{e} V_{\mathrm{n}} r_{\mathrm{n}} \sim n h / 2 \pi$ can be expressed as an equality through the mediation of an arbitrary constant multiplier $\boldsymbol{\kappa}$ :

$$
m_{e} V_{\mathrm{n}} r_{\mathrm{n}}=\kappa n h / 2 \pi \quad==>\quad V_{\mathrm{n}}=\kappa n h / 2 \pi m_{e} r_{\mathrm{n}}
$$

Based on (11) and (12) the expression for $r_{\mathrm{n}}$ can be derived:

$$
r_{\mathrm{n}}=\kappa n^{2} h^{2} \varepsilon_{0} / e^{2} \pi m_{e^{*}}
$$

Since in accordance with (13) on condition that $n=1 \kappa h^{2} \varepsilon_{0} / e^{2} \pi m_{e}=r_{1}$, the expression (13) can be re-written as

$$
r_{\mathrm{n}}=r_{1} n^{2}
$$

Through the substitution (14) into (12) the expression for $V_{\mathrm{n}}$ takes the form:

$$
V_{\mathrm{n}}=\boldsymbol{\kappa} h / 2 \pi m_{e} r_{1} n
$$

Since in accordance with (15) on condition that $n=1 \kappa h / 2 \pi m_{e} r_{1}=V_{1}$, the expression (13) can be re-written as

$$
V_{\mathrm{n}}=V_{1} / n \text {. }
$$

The electron's rotation period at the stationary orbit with ordinal number $n$ is expressed by the formula:

$$
T_{\mathrm{n}}=2 \pi r_{\mathrm{n}} / V_{\mathrm{n}}
$$

Through the substitution (14) and (16) into (17) the expression for $T_{\mathrm{n}}$ takes the form:

$$
T_{\mathrm{n}}=2 \pi r_{1} n^{2} /\left(V_{1} / n\right)=2 \pi r_{1} n^{3} / V_{1}
$$

Since in accordance with (18) on condition that $n=12 \pi r_{1} / V_{1}=T_{1}$, the expression (13) can be re-written as

$$
T_{\mathrm{n}}=T_{1} n^{3}
$$

Since a frequency is in inverse proportion to a period $(v=1 / T)$, the expression for frequencies has the form:

$$
v_{\mathrm{n}}=v_{1} / n^{3}
$$

Note that in accordance with (20) the frequency $\boldsymbol{v}_{\mathbf{n}}$ is in inverse proportion to $\boldsymbol{n}^{3}$, whereas the frequencies (4) given by Rydberg's formula (3) are in inverse proportion to $\boldsymbol{n}^{2}$. This problem is resolving by taking into account that integer number of periods of de Broglie's wave [3], [8] should be fitted in a stationary orbit. It seems likely that the periods of de Broglie's wave (not the electron itself) are responsible 
for associated frequency. Because of this the effective frequency $v^{\prime}{ }_{n}$ produced by $n$ periods of de Broglie's wave disposed along the stationary orbit number $n$ will be $n$ times greater than frequency of electron's rotation at this orbit:

$$
v_{\mathrm{n}}^{\prime}=n \quad v_{\mathrm{n}}=v_{1} / n^{2}
$$

\section{Some Consequences}

\subsection{Modified Quantization Rule}

Instead of (9) the more general quantization rule (22) is in use in our model:

$$
m_{e} V_{\mathrm{n}}^{*} r_{\mathrm{n}}^{*}=\kappa n h / 2 \pi,
$$

where $r_{\mathrm{n}}^{*}$ - radius of the stationary orbit having number $n$ and $V_{\mathrm{n}}^{*}$ - velocity of the electron at that orbit in accordance with our model. Now it is possible to figure out the value for the coefficient $\boldsymbol{\kappa}$ based on the ratio of our first stationary orbit radius $(0.840 \AA)$ to Bohr's radius $(0.529 \AA)$.

First, let us divide (22) by (9) and take the square:

$$
\left(V_{\mathrm{n}}^{*} r_{\mathrm{n}}^{*}\right)^{2} /\left(V_{\mathrm{n}} r_{\mathrm{n}}\right)^{2}=\boldsymbol{\kappa}^{2}
$$

Second, let us derive the expression for $\left(V_{1} r_{1}\right)^{2}$ from (5):

$$
\left(V_{1} r_{1}\right)^{2}=e^{2} r_{1} / 4 \pi \varepsilon_{0} m_{e^{*}}
$$

The expression for $\left(V_{1}^{*} r_{1}^{*}\right)^{2}$ has the similar form:

$$
\left(V_{1}^{*} r_{1}^{*}\right)^{2}=e^{2} r_{1}^{*} / 4 \pi \varepsilon_{0} m_{e^{*}}
$$

By dividing (25) by (24) we obtain the following proportion:

$$
\left(V_{1}^{*} r_{1}^{*}\right)^{2} /\left(V_{1} r_{1}\right)^{2}=r_{1}^{*} / r_{1}
$$

It can be re-written, in view of (13) for the case $n=1$, as

$$
r_{1}^{*} / r_{1}=\boldsymbol{\kappa}^{2},
$$

where $r_{1}=0.529 \AA$ - Borhr's radius, $r_{1}^{*}=0.840 \AA$ - the radius of the first stationary orbit in our model.

Therefore, the coefficient $\kappa^{2}$ is equal to $0.840 / 0.529 \approx 1.588$. Since the value 1.588 is very close to $\sqrt[3]{2^{2}}$, we can write $\boldsymbol{\kappa}^{2}=\sqrt[3]{2^{2}}, \quad \boldsymbol{\kappa}=\sqrt[3]{2}$ and, as a result, our quantization rule (12) takes the form:

$$
m_{e} V_{\mathrm{n}}^{*} r_{\mathrm{n}}^{*}=\sqrt[3]{2} n h / 2 \pi
$$

It is equal to the statement that the length of the circumference of the orbit having number $n$ is an integer number $n$ of the modified de Broglie's wavelength:

$$
\lambda_{\mathrm{n}}^{*}=\sqrt[3]{2} h / m_{e} V_{\mathrm{n}}^{*} .
$$




\subsection{Amount of Energy Emitted as a Quanta}

While the hydrogen atom transformed from one stationary state characterized by the orbit radius $r_{\mathrm{m}}$ $(m>n)$ into another stationary state characterized by the orbit radius $r_{\mathrm{n}}$, its energy changed from $E_{\mathrm{m}}$ to $E_{\mathrm{n}}$ and a quanta of energy $\Delta E_{\mathrm{m} n}=E_{\mathrm{m}}-E_{\mathrm{n}}$ is emitted. In accordance with Bohr's model $\Delta E_{\mathrm{m} n}=h v_{\mathrm{mn}}$, where the frequency $v_{\mathrm{m} n}$ is expressed by Rygberg's formula (3). Note, that the above formula is no more than the second Bohr's postulate [2]-[4].

Energy of the hydrogen atom is expressed by the formula $E=-\left(1 / 4 \pi \varepsilon_{0}\right)\left(e^{2} / 2 r\right)$, where $r$ - radius of electron's orbit. In other words, energy is in inverse proportion to the radius. Since radiuses of electron's orbits in our model are greater by a factor of $\boldsymbol{\kappa}^{2}$ than the radiuses in Bohr's model, energies of the hydrogen atom at all stationary states (and differences of these energies as well) will be less than energies in Bohr's model by a factor of $\boldsymbol{\kappa}^{2}$. To be compatible with our model, Bohr's formula for energy emitted by the atom as quanta shall be transformed into (30):

$$
\Delta E_{\mathrm{mn}}^{*}=h v_{\mathrm{mn}} / \kappa^{2}
$$

To put it otherwise, the values $v_{\mathrm{m} n}$ are given by Rydberg's formula (they come from experiment and shall be considered as physical constants), $h$ is the constant as well. To express the less energy emitted by atom in our model due to greater radiuses of stationary orbits through the mediation of the constants $h$ and $v_{\mathrm{m} n}$, the product $h v_{\mathrm{m} \mathrm{n}}$ has to be divided by $\boldsymbol{\kappa}^{2}$.

\section{The Essence of the Discrepancy between Suggested Model and Bohr's Model}

Starting from his well-known pair of postulates:

$$
m_{e} V_{\mathrm{n}} r_{\mathrm{n}}=n h / 2 \pi \quad \text { and } \quad \Delta E_{\mathrm{mn}}=E_{\mathrm{m}}-E_{\mathrm{n}}=h v_{\mathrm{mn}},
$$

N. Bohr came back to Rydberg's formula and expressed Rynberg's constant through the mediation of some other fundamental constants:

$$
R=m_{e} e^{4} / 8 \varepsilon_{0}^{2} c h^{3} .
$$

$\mathrm{N}$. Bohr considered this result as an evidence for the validity of his approach, whereby the value for the radius of the ground stationary orbit is equal to $0.529 \AA$.

In contrast to this, while started immediately from Rydberg's formula, we arrived at the different value $(0.840 \AA)$ for the radius of the ground stationary orbit. Following Bohr's way it is possible to derive this value for the radius as well but with the use of the modified postulates:

$$
m_{e} V_{\mathrm{n}} r_{\mathrm{n}}=\sqrt[3]{2} n h / 2 \pi \quad \text { and } \quad \Delta E_{\mathrm{mn}}=h v_{\mathrm{mn}} / \sqrt[3]{2^{2}}
$$

Why the postulates (31) and (33), being both in good agreement with Rydberg's formula, lead to different values for the radius of the ground stationary orbit? The answer resides in the fact that Rydberg's formula can always be derived from the parametric "postulates":

$$
m_{e} V_{\mathrm{n}} r_{\mathrm{n}}=\kappa n h / 2 \pi \text { and } \Delta E_{\mathrm{mn}}=h v_{\mathrm{mn}} / \kappa^{2},
$$

and no matter what value is assigned to the parameter $\boldsymbol{\kappa}$. 
An appropriate value for the parameter $\boldsymbol{\kappa}$ has to be chosen based on several physical grounds. In our model the value of $\sqrt[3]{2}$ for the parameter $\boldsymbol{\kappa}$ arises as a result of a very natural assumption that the simple relation between the motion of the electron in the atom and the radiation emitted exists and that $v_{1}=R c$ is the frequency of electron's rotation at the ground stationary orbit of the hydrogen atom.

Let us show that Rydberg's formula (3) can always be derived from the parametric "postulates" (34), and not just from Bohr's postulates (31). Note, that (31) can be obtained from (34) on condition that $\boldsymbol{\kappa}=1$.

At any stationary orbit electrostatic force is balanced by centrifugal force:

$$
m_{e} V_{\mathrm{n}}^{2} / r_{\mathrm{n}}=e^{2} / 4 \pi \varepsilon_{0} r_{\mathrm{n}}^{2} .
$$

Substituting the quantization rule taken from the parametric "postulates" (left side of (34)) in (35) we can get an expression for the radius of the stationary orbit number $n$ :

$$
r_{\mathrm{n}}=\boldsymbol{\kappa}^{2} \varepsilon_{0} n^{2} h^{2} / m_{e} e^{2} \pi
$$

By taking into account (36), the total energy of the electron (kinetic energy plus potential energy) at the stationary orbit number $n$ can be expressed by

$$
E_{\mathrm{n}}=-\left(1 / 4 \pi \varepsilon_{0}\right)\left(e^{2} / 2 r_{\mathrm{n}}\right)=-m_{e} e^{4} / 8 \varepsilon_{0}^{2} \boldsymbol{\kappa}^{2} n^{2} h^{2} .
$$

Now the energy of the quanta as a difference of the total energy at stationary orbit number $m$ and $n$ is expressed by

$$
\Delta E_{\mathrm{mn}}=E_{\mathrm{m}}-E_{\mathrm{n}}=m_{e} e^{4} / 8 \varepsilon_{0}^{2} \boldsymbol{\kappa}^{2} h^{2}\left(1 / n^{2}-1 / m^{2}\right) .
$$

Turning from energy to frequency based on the equation $\Delta E_{\mathrm{m} n}=h v_{\mathrm{m} n} / \boldsymbol{\kappa}^{2}$ of the parametric "postulates" (right side of 34), we obtain

$$
\begin{gathered}
h v_{\mathrm{mn}} / \boldsymbol{\kappa}^{2}=m_{e} e^{4} / 8 \varepsilon_{0}^{2} \boldsymbol{\kappa}^{2} h^{2}\left(1 / n^{2}-1 / m^{2}\right), \\
v_{\mathrm{mn}}=m_{e} e^{4} / 8 \varepsilon_{0}^{2} h^{3}\left(1 / n^{2}-1 / m^{2}\right) .
\end{gathered}
$$

Note that, while turning from (38) to (39) and (40), the $\boldsymbol{\kappa}$-dependence is disappeared.

The expression (40) is nothing but Rydberg's formula (3) where $R c=m_{e} e^{4} / 8 \varepsilon_{0}^{2} h^{3}$.

\section{Conclusion}

In contrast to N. Bohr postulate: "... frequency $v$ has no simple relation to the motion of the particles of the atom, but is given by the relation $h v=E^{\prime}-E^{\prime \prime}, \ldots$.. [9], it is suggested to consider the frequency of emitted radiation as a difference of two effective frequencies (11) closely related to electron's rotation at two stationary orbits. The differences of this kind are explicitly expressed by Rydberg's formula comprising well-verified hydrogen spectrum data. Note that the hydrogen atom can be considered as a frequency mixer that is similar to radio-frequency mixer of a supergeterodyne receiver.

The given approach provides the more natural and more understandable model. Thus, one piece of the hydrogen atom puzzle falls into place and the model is coming to less mystery because it gives the simple 
relation between the frequency of the emitted radiation and frequencies of electron rotation.

According to the suggested model, the radius of electron orbit at ground stationary state of the hydrogen atom is bound to be greater than Bohr's radius by a factor of $\sqrt[3]{2^{2}} \approx 1.588$. The same result can be derived based on Bohr's model but under the assumption that $m_{e} V_{\mathrm{n}} r_{\mathrm{n}}=\sqrt[3]{2} n h / 2 \pi$ instead of weak-founded Bohr's postulate $m_{e} V_{\mathrm{n}} r_{\mathrm{n}}=n h / 2 \pi$, that gave him no way of revealing the relation between the electron motion and frequency emitted.

In summary, the Bohr's model of the hydrogen atom was undoubtedly as a step forward in our understanding of the structure of the matter. Nevertheless, it gives wrong numerical values for the parameters of the hydrogen atom as a consequence of the failure to take in account the starting conditions.

The main contribution of the paper:

1) It is shown (Paragraph 7) that Rydberg's formula can be derived from the more general (than Bohr used in his model) parametric postulates (24) with no matter what value the parameter $\boldsymbol{\kappa}$ has. This points to an inaccuracy of Niels Bohr model of the hydrogen atom because it is not clear why preference is given to the value $\boldsymbol{\kappa}=1$ in Bohr's model.

2) To be well-grounded in deciding on a value of the parameter $\boldsymbol{\kappa}$ some physical considerations due to be taken into account. It is suggested (Paragraph 2) to consider Rydberg's formula (3) as an explicit relation between frequencies of electron's rotation in the atom and frequency emitted. In other words, it is suggested to consider that Rydberg's formula (3) expresses the frequency of emitted radiation as a difference (4) of two frequencies that are closely related to the frequencies of electron's rotation at the corresponding stationary orbits. As a result, the value $R c$ is considered as the frequency of electron's rotation at the ground stationary orbit. It allows us to figure out (Paragraph 3 ) a new value $(0.840 \AA$ ) for the radius of the ground stationary orbit in the hydrogen atom.

\section{References}

[1] Rutherford, E. (1911). The scattering of $\alpha$ and $\beta$ particles by matter and the structure of the atom. Philosophical Magazine, 21, 669-688.

[2] Bohr, N. (1913). On the constitution of atoms and molecules. Philosophical Magazine, 26(151), 1-24.

[3] Gautreau, R., \& Savin, W. (1999). Schaum's Outline of Modern Physics (2 ${ }^{\text {nd }}$ ed.). New York: McGraw-Hill.

[4] Derivation of Bohr's model for hydrogen spectrum. Retrieved August 23, 2014, from the Physics LAB website: http://dev.physicslab.org/AtomicNuclear_BohrModelDerivation.xml

[5] Balmer, J. J. (1885). Annalen der Physik, 261, 80.

[6] Lyman, T. (1906). Astrophysical Journal, 23, 181.

[7] Mohr, P. J., Taylor, B. N., \& Newell, D. B. (2006). CODATA recommended values of the fundamental physical constants. Reviews of Modern Physics, 80(2), 633-730.

[8] Broglie, L. (1946). Matter and Light. New York: Dover.

[9] Bohr, N. (1922). The Structure of the Atom (Nobel Lecture).

V. Tomashau was born in Russia (Sakhalin Island) in 1947. He was graduated from Tomsk State University (Tomsk, Russia) in 1970 with the degree in radio-physics and electronics. He received his PhD degree in computer science from Institute of Cybernetics of the National Academy of Science of Ukraine (Kiev) in 1991. After the graduation from the university, he worked in Tomsk and, from 1972, in Minsk (Belarus) where he dealt with software development for logic design in microelectronics, compiler construction, 
VHDL programming and simulation. From 2007, he worked in LC Engineers, Inc., Rahway, New Jersey, USA. His research interests include logic circuit design and optimization, the fundamentals of atom structure, energy emission and absorption by the atom. 